\title{
Using cannabis for pain management after spinal cord injury: a qualitative study
}

\author{
John A. Bourke ${ }^{1,2} \cdot$ Victoria J. Catherwood ${ }^{3} \cdot$ Joanne L. Nunnerley $\mathbb{1}^{1,4} \cdot$ Rachelle A. Martin $\mathbb{1}^{1,5} \cdot$ \\ William M. M. Levack ${ }^{5} \cdot$ Bronwyn L. Thompson $^{4} \cdot$ Richard H. Acland $^{1}$
}

Received: 4 August 2019 / Accepted: 12 September 2019

(c) The Author(s), under exclusive licence to International Spinal Cord Society 2019

\begin{abstract}
Study design A descriptive qualitative study.

Objectives To explore why individuals with spinal cord injury (SCI) choose to use cannabis to manage their pain and their experiences in doing so.

Setting Community-dwelling adults with SCI in New Zealand.

Methods Semi-structured interviews were conducted with individuals who had a SCI, experienced pain, and self-reported use of cannabis to manage their pain. Interviews were recorded, transcribed, and subject to thematic analysis.

Results Eight individuals participated in this study. We interpreted six themes that captured the participants' perspectives regarding their choice to, and perceptions of, using cannabis to manage SCI pain. Participants were motivated to use cannabis when other pain management strategies had been ineffective and were well-informed, knowledgeable cannabis consumers. Participants reported cannabis reduced their pain quickly and enabled them to engage in activities of daily living and participate in life roles without the drowsiness of traditional prescribed pain medication. Despite the positive aspects, participants were concerned about the irregularity of supply and inconsistent dosage.

Conclusions Findings show that cannabis is used to reduce pain after SCI and enable increased community participation. Findings suggest that future studies examining the efficacy of cannabinoids in managing pain include function and participation outcome measures rather than solely focusing on measuring pain intensity. Focusing on meaningful outcomes may contribute to a greater understanding of the experiences of people with SCI.
\end{abstract}

\section{Introduction}

Spinal cord injury (SCI) has a number of secondary complications, which can continue throughout a person's life [1]. One of the most common and debilitating complications is

John A. Bourke

johnny.bourke@burwood.org.nz

1 Burwood Academy of Independent Living, Christchurch, New Zealand

2 Menzies Health Institute, Griffith University, Queensland, Australia

3 School of Medicine, University of Otago, Christchurch, New Zealand

4 Orthopaedic Surgery and Musculoskeletal Medicine, University of Otago, Christchurch, New Zealand

5 Rehabilitation Teaching and Research Unit, Department of Medicine, University of Otago, Wellington, New Zealand severe and chronic pain $[1,2]$. The prevalence of chronic pain after SCI is estimated to be between 66 and 79\% [3]. Chronic SCI-pain can interfere with activities of daily living, mood and quality of life [3-5]. Furthermore, few management options provide adequate pain relief for chronic pain [3]. There is low level evidence supporting the use of cannabinoids as a treatment for pain in chronic noncancer conditions [6-8]. In this paper we use the term 'cannabis', which refers to the use of cannabis plants and plant material for medical reasons which was not prescribed by a physican $[9,10]$. Whole plant cannabis has been shown to have a reasonably safe pharmacological profile [11], and can include the psychotropic cannabinoid delta-9tetrahydrocannabinol (THC) and the non-psychotropic cannabinoid cannabidiol (CBD) [12].

Internationally, increasing research is beginning to explore the use and potential therapeutic effects of cannabis for individuals with SCI [13-15]. While legal access to cannabis varies throughout the world, the New 
Zealand government passed a Misuse of Drugs (Medicinal Cannabis) Amendment Act in 2018, setting a Medicinal Cannabis Scheme to commence in early 2020 [16]. Certain medical cannabis products are available on prescription in New Zealand, however this route is a timeconsuming, expensive process and excludes prescription use of cannabis plants. In the 2012/2013 New Zealand Health Survey, $11 \%$ of adults $(397,000)$ reported using cannabis in the last 12 months, with $42 \%(167,000)$ of these individuals reporting they used cannabis for medicinal purposes [17].

It is critical that clinicians, researchers, and individuals who may choose to use cannabis are informed of the benefits - and risks - of using cannabis to manage their pain. Research exploring the use of cannabis to treat non-cancer chronic pain is still in its infancy with the findings, and opinions, being inconsistent and polarising [10, 18-22]. Research exploring the user perspectives of cannabis to manage pain following SCI almost always utilise questionnaires [13-15, 23, 24], apart from one focus group conducted by Hawley et al. [13]. Few in-depth qualitative research exploring user perspectives are available. The perspectives of individuals with SCI who use cannabis for their pain, should contribute to the clinical, social, and political discussion. The aim of this study was to understand why individuals with SCI choose to use cannabis to manage their pain and their experiences of doing so.

\section{Methods}

\section{Design}

This study involved qualitative descriptive analysis of anonymous, semi-structured interviews with adults who used cannabis for pain after SCI. The University of Otago Human Ethics Committee provided ethics approval for this study (H18/081).

\section{Participant recruitment}

Participants had to self-identify as using cannabis for pain associated with their SCI. Participants were required to be 18 years of age or over, reside in New Zealand, and be able to communicate in English. Participants were excluded if they had any comorbid conditions inhibiting communication and participation in interviews. Snowball recruitment was used, starting with personal contacts in the SCI community [25]. Once contacted, all participants were asked to forward the lead researcher's (JN) contact details to any acquaintances they might know who used cannabis to manage pain. Potential participants were advised to phone the lead researcher $(\mathrm{JN})$.
Due to the illegal status of cannabis in New Zealand it was essential to maintain participant confidentiality and anonymity. Participants were all asked to provide pseudonyms from first contact, and all subsequent interactions and documentation used the participants' pseudonym, so their real name was not recorded anywhere. Participants were able to access information and consent forms from the research institute's website to prevent emailing or posting forms to participants. At first contact, the information and consent sheets were also read to the participants by phone, and the confidentiality process of the research was also explained to them. All participants provided verbal consent to take part in the study.

\section{Data collection}

Participants were invited to contribute to an interview, either in person at a location of their choosing or by internet-based videoconference. Two researchers (JB and VC) conducted the interviews, which were audio recorded and transcribed. Field notes were completed after each interview. Interview questions were informed from the literature, as well as clinical and consumer members on the research team. Questions were ordered from the broad (e.g. Can you tell us about your experience of pain?) to the more specific (e.g. What percentage of THC and CBD were you using?). For all participants, we also collected basic demographic information (e.g. age, gender and spinal injury level).

\section{Data analysis}

Interview transcripts were subject to thematic analysis-a flexible approach that can be used to identify, analyse, and report patterns within data [26, 27]. The six stages of thematic analysis suggested by Braun and Clarke [26] were used, and data were analysed by hand. First, all transcripts were read by the team to familiarise themselves with the data. Second, two researchers (JB and VC) created initial higher-level codes (aspects in the data that reflected a single concept). Third, similar and related codes were organised into broader patterns through visual examination and became candidate themes. Themes were refined in discussions with the entire research team. Fourth, these themes were supported by illustrative quotes from the interview data. The integrated narrative was discussed by all authors to verify the coherence within and between themes, and the research question. At this point the candidate themes were finalised. Lastly, a report was written explaining each theme with examples ensuring a coherent flow connecting all themes. The non-italicised quote text between parentheses is an explanatory addition to enable clearer understanding. 
Analytic rigour was pursued in several ways. First, researchers regularly referred to the original interview transcripts to ensure their analysis was connected to the participants' voice. Second, theme development was discussed at each team meeting and regularly via email. Third, a complete record of the data collection and data analysis process was kept so that an independent researcher could audit the research process. Finally, the research team included a person with SCI, rehabilitation clinicians, a pain researcher, a pain consultant, a medical doctor, and an adviser with the lived experience of SCI of using cannabis to manage pain. The researchers conducting the interviews had no prior relationship with research participants.

\section{Results}

The study sample consisted of eight participants, (two woman, six men) —an appropriate number for an exploratory, qualitative study of this nature [27]. Table 1 gives a summary of the participants' demographic characteristics. Three participants were interviewed in person and five were interviewed via videoconference. Interviews ranged between 35 and $60 \mathrm{~min}$.

Six themes were derived to capture the participants' perceptions of using cannabis to manage their pain.

Table 1 Participant demographic characteristics

\begin{tabular}{ll}
\hline Characteristic & $N(\%)$ \\
\hline Age (years) & $1(13)$ \\
$20-39$ & $5(62)$ \\
$40-59$ & $2(25)$ \\
$60+$ & \\
Gender & $6(75)$ \\
Male & $2(25)$ \\
Female & \\
Ethnicity & $5(62)$ \\
NZ European & $2(25)$ \\
NZ Māori & $1(13)$ \\
Other & \\
SCI level & $1(13)$ \\
C1-C4 & $5(62)$ \\
C5-C8 & $2(25)$ \\
T1-S5 & \\
Method of cannabis use & $5(62)$ \\
Edibles & $3(38)$ \\
Smoking &
\end{tabular}

\section{Theme one: the prison of pain}

Participants reported living in unbearable pain. This experience was akin to feeling trapped in a prison: there was an overwhelming feeling of loss of control over one's situation and choices:

It just takes over your, your life. I mean when you have the pain you can't do anything, you know? You can barely talk, um, and, um, it just, the pain just overtakes everything. (Participant 6)

Feeling trapped by pain inhibited the participants' ability to engage in meaningful activities of daily life, family interactions, social situations, or employment. The allconsuming nature of pain eclipsed all other experience until the pain subsided:

I just have to stop and learn to breathe and just sit there and do nothing and just hopefully that, um, eases off basically. (Participant 4)

Finding ways to manage and reduce pain appears to be a priority. Participants often referred to trying a variety of pain management strategies with minimal success:

Since day dot I've tried everything and you know nothing has seemed to work. (Participant 1)

The most common participant management strategy reported for their pain was prescription medication, which presented certain drawbacks.

\section{Theme two: the (prescribed) drugs do not work}

The participants described multiple attempts to reduce pain through prescription medication strategies. However, they reported these strategies were often ineffective, had detrimental side-effects, or both. The most commonly reported side-effects were drowsiness, fatigue and constipation. Several participants referred to prescription medication putting them into a 'zombie-like state':

All of the prescription medications are generally opiate or neuroleptic based medications like gabapentin or opiate based, things like oxycodone, fentanyl patch, that sort of stuff, so all of them, I think, the main thing's I find they make me drowsy and they do impair my cognitive ability to think clearly and, and be fully compos mentis. (Participant 7)

Many participants expressed their dislike at having to live in a constant state of fatigue and drowsiness, and that 
they were unable to engage in the activities important to them:

It dulls the pain but then I'm sleepy and, um, yeah, just dozy and feel like a bit of a zombie, I dunno. And I don't really like it, that's not the way I wanna live really. (Participant 4)

\section{Theme three: choosing to use cannabis}

In Theme three we describe why participants chose to use cannabis, and more specifically how and why they chose the particular type of cannabis product. Participants' reported using cannabis products because the prescribed pain medication was ineffective and/or the side-effects caused frustration and exhaustion. When making the decision to use cannabis products one participant (who trialled a high $\mathrm{CBD} /$ low THC oil) reflected:

Well look, I've got nothing to lose to try it and I'm not getting high from it so, what have I got to lose? And it, it does a better job than the stuff that's legal. (Participant 4)

With no educated health professional to guide administration, participants reported having to trial products within an unregulated, and often unfamiliar, environment. Participants described themselves as well-informed consumers of cannabis products over time, with different products creating unique subjective effects. Several participants reported wanting something that effectively managed pain but did not create dysphoria which had a detrimental effect on participants' mind and ability to participate within the community:

You can get paranoid, the rest of it, and your body still relaxed but your mind is not quite with it... so I don't like that side of it, that's what I hate, I hate not feeling in control... Like if I was to smoke it I wouldn't want to go out into the community. (Participant 1)

Participants reported a fundamental difference between being 'high', and effectively managing one's pain.:

I'm not wanting to get stoned all day, every day because that's not me, but I get to live every day.

There's a big difference. (Participant 3)

Participants did not want to replace the detrimental side effects of prescription medication (drowsiness) with equally unhelpful side effects of cannabis (dysphoria). With no objective testing for the THC levels in a product, assessing its effect was subjective:

There's some [high CBD cannabis] out there that'll relax your body, which is the one that helps me relax my mind, get out of that state of pain, or there's the one that's makes you go to sleep [high THC]. Those are the two simple breeds really. I'm fortunate sometimes to get the one that makes me live through the day and not sleep through the day. (Participant 5)

As such, personal experimentation, research through online forums and groups, and discussion with peers all contributed to determining products that worked for them. One participant described experimenting with a variety of methods and monitoring changes in their pain levels:

I started to eat it raw. The leaves, the stalks, I started chopping it up and putting it into my salads and things like that. Um, I don't know exactly what it was doing then, but the pain would come and go, and the burning sensations were all starting to change, and with a mixture of eating it, the stalks, the leaves and smoking, um, it, the pain started to subside. (Participant 2)

\section{Theme four: negotiating an unfamiliar illegal context}

There are few legal cannabis products on the market in New Zealand and all participants were aware of these. However, only one participant was using a legal product. Most participants considered legal cannabis-based medication to be unaffordable with access limited by what they perceived as a cumbersome level of bureaucracy and administration processes:

Why spend a fortune going back to your doctor when I can grow something out in my backyard that would do the same thing... why go to a doctor when all they're going to do is refer you on and then it's going to be eight months down the track before you know. (Participant 3)

Despite the uncertainty, obtaining an illegal product was considered easier, cheaper, and as effective for managing their pain as prescribed products. However, participants described blurred lines between the illegality of cannabis and the negative perceptions that influenced their experience of using cannabis. For many participants, using cannabis was initially something morally wrong, or questionable: 
I come from a family where I, yah know, we've, my parents taught me a few morals and, with right and wrong, I mean, um, I was never really into cannabis and just for taking it for getting high. (Participant 4)

Several participants described that their use caused them to review their view of on cannabis in society:

Oh in my former life, if someone mentioned cannabis I would have thrown my arms up in horror. I think this is a last resort because you're always waiting to see what will help (Participant 6).

The illegal nature of cannabis products meant participants hid their use to avoid repercussions with the law. One participant described how legalisation would reduce the stress:

Just to make it legal so we don't have to look over our shoulders all the time and think about what's gonna happen and consequences. (Participant 5)

Interactions with health professionals were strongly influenced by the illegal nature and stigma of cannabis. Participants reported both positive and negative interactions, with the positive being characterised by participants as a subtle acceptance more than validation or approval. Participants also mentioned a lack of knowledge amongst clinicians, instead seeking information through their close contacts or suppliers who had a high degree of experiential knowledge:

[my supplier] has a ton of information, and connections just keep talking and just working with the cannabis oils and things like that... what to do and how to mix it. (Participant 2)

There were also many instances of resistance from health professionals who often alluded to the lack of convincing evidence surrounding the safety and effectiveness of cannabis products:

The pain specialists don't, won't, I found, endorse a marijuana-based product because they, well their reasoning to me was that there's not enough evidence-based research yet to warrant the use of it. (Participant 7)

Once participants chose to use cannabis, and despite having to negotiate an often unfamiliar illegal context, almost all participants described cannabis effectively helping them to the extent they felt less disabled and could participate in the community again.

\section{Theme five: free to pursue meaningful outcomes}

Overall, participants described cannabis as an effective pain management strategy, and in a sense 'freed' participants from their 'prison of pain' (Theme one):

it's helped my whole health and helped the pain everything, my mental pain, physical pain the whole lot you know, ever since I've been on it. (Participant 2)

Participants reported a variety of potential ways in which this occurred, from near-complete pain reduction, to making the experience of pain more tolerable. Whatever the mechanism of effective pain management, the ultimate flow-on effect was to improve participants' functional and participation outcomes. For example, functionally, cannabis did not create the fatigue and drowsiness of prescribed medication, and helped to improve the sleep experienced by many participants:

It just made me sleep, it gave me a deeper sleep, I guess, so I just was less disturbed by my pain when I was sleeping. (Participant 7)

Cannabis also improved participants' quality of life and facilitated a renewed capacity to participate more in life, such as spending time with family:

The main down sides are, as I said, I find it hard to, to really pinpoint anything but the benefits, it, it's, um, just a happier life, um, I'm not getting any younger, I mean I'm 56, 57 soon, um, and I just want to enjoy life and it seems to really help to do those things and enjoy my family, yah know? (Participant 4)

Family were often able to see the beneficial effects of using cannabis products which in turn reduce the family member's own discomfort of seeing a loved one in pain:

My whole family, um, don't even care where I smoke weed. It's not because I smoke weed, it's because they've seen the effects. They've seen the effects when I'm in pain, they, they cry. But compared to when I take weed, it's, it's totally different. (Participant 3)

Participants described many positive implications of using cannabis for pain, highlighting a range of meaningful outcomes on quality of life, sleep, and community participation. 


\section{Theme six: you cannot always get what you want}

Participants repeatedly described unpredictability, inconsistency, and a lack of information associated with an unregulated market supply of cannabis products. Ambiguity and variability surrounded participants' understanding of the cannabinoid composition of cannabis used (e.g. CBD/ THC ratio), interactions with prescribed medications, and having access to a regular and consistent supply, as one participant remarked:

[my suppliers] live in isolated areas, you can't get a hold of them by cell phone or anything like that, it's only when they might come into town and I might receive a message (Participant 6 )

Participants were also seeking a consistent strength or dose, and to know how much they should be taking for predictable pain relief. However, in an illegal context their ideas, concerns and expectations were not always achievable. Participants often alluded to not knowing what they're buying, and getting the incorrect level of THC:

If you're not going to grow it yourself, you're then therefore left to buy it. Now when you buy it, you don't know what you're getting, you don't know what strain it is, you don't know what product it is. I've had instances where I've bought the wrong product. Where's, where's the fairness in that? (Participant 3)

\section{Discussion}

This study used individual interview data to explore the use of cannabis to manage pain in individuals with SCI. For the participants in this research, cannabis was a broadly positive and useful intervention for managing SCI pain. Several surveys have also reported that cannabis has a positive effect on managing pain in SCI $[13,15,24]$. In a survey of individuals with SCI in Denmark, $(n=537), 59 \%$ reported a positive effect on pain and spasticity [24]. Likewise, a survey conducted in Colorado, USA, reported 59\% of participants $(n=27)$ endorsed the use of cannabis specifically for its SCI pain reduction qualities [13].

Participants in our study were motivated to use cannabis, often as a last resort, when other pain management strategies were ineffective or the negative side-effects of prescribed medication, such as drowsiness, fatigue, and of being in a dysphoric 'zombie' state, became intolerable. Participants in our study found these side effects limited basic communication and their ability to focus, whereas using cannabis helped without these side-effects. Similar findings were identified in qualitative analysis of a single focus group in the USA where participants preferred using cannabis over prescribed medications as cannabis had fewer negative side-effects, and cannabis use had enabled their participants to discontinue narcotic and anti-anxiety medication [13]. Our participants reported increased cognitive clarity associated with reducing their prescription medications, enabling them to feel they could engage in activities and with social connections. Furthermore, for many participants in this study, cannabis use reduced the use of prescribed medications, and a feeling of overall improvement in physical health, with fewer secondary complications (e.g. fatigue, constipation, and sleep disruption).

This study shows that for our participants cannabis produced important and meaningful outcomes along with reducing pain. Campbell et al. [10]. highlight that research examining the efficacy of cannabinoids in managing pain has traditionally focused on a change in pain scores rather than examining clinically meaningful outcomes of function and participation. Our research supports the suggestion that cannabis might not eliminate pain intensity per se, but instead enables those with pain to tolerate it more effectively [28]. Participants in our study described improved sleep, and increased participation in family, and community life-outcomes which might contribute to any reduced pain scores [29]. The contribution of qualitative research and lived experience provides unique insights, particularly during cannabis health policy development

Our research highlighted the unpredictability of accessing unregulated illegal products with irregularity of supply, inconsistent THC/CBD dosage and potentially facing criminal consequences. Users' concern about the legality of cannabis have been raised in several studies [14, 28], although the legal status of cannabis is under increased debate in many jurisdictions. Many countries are implementing medicinal cannabis schemes in order to regulate and protect the quality of products, continuity of supply, and reduce criminal prosecution or unregulated high prices for patients.

In the absence of professional advice, participants relied on their own research to determine dosage and chemical make-up of cannabis. This places people at risk of adverse effects of cannabis use such as interactions with pharmaceutical medications. An international survey of clinicians [30] who care for individuals with SCI $(n=153)$ reported that $52 \%$ of clinicians believe they should be able to offer patients cannabis, yet only $13 \%$ were employed by a facility who prescribed medicinal cannabis, suggesting a discrepancy between clinicians interest in medicinal cannabis and policy. As more Medicinal Cannabis Schemes are introduced it is critical that clinicians have adequate education about medicinal cannabis products, and the benefits 
and risks, in order to provide informed advice to their patients.

The aim of this study was not to generalise, but to provide sufficient detail regarding the participant group, the context in which the research occurred, and the processes involved in the data analysis so readers can decide if results are relevent to other settings [31, 32]. Eight participants is considered sufficient for inductive thematic analysis, large enough for identifying patterns across cases, yet small enough to focus on individual experiences [27]. It is plausible that only people with a positive experience of using cannabis participated in the study. Future qualitative research would benefit from exploring the perspectives of those for whom cannabis might not help. As participants in our study had no certainty over THC/CBD ratios it is impossible to determine which constituents within the participant's cannabis helped.

This study highlights how cannabis can be used for pain after SCI to enable increased social and community participation. Individuals were well informed about products and reported seeking the pain alleviating effects of cannabis, while actively avoiding the undesirable psychotropic effects. As cannabis is being more widely considered for medicinal use, larger studies on cannabis for SCI pain, particularly user perspectives, are warranted to inform clinical practice in pain and SCI. Efficacy studies should consider including measures of function and participation to ensure greater understanding of the experiences of people with SCI, rather than solely pain intensity measures.

Acknowledgements The research team would like to thank the person with SCI who advised on aspects of this study.

Author contributions All authors apart from WM were involved in the study design. JN, JB and VC were responsible for the data collection. All authors were involved in the data analysis. JB was responsible for the first draft the paper and all authors contributed to further edits and iterations of the paper.

\section{Compliance with ethical standards}

Conflict of interest The authors declare that they have no conflict of interest.

Publisher's note Springer Nature remains neutral with regard to jurisdictional claims in published maps and institutional affiliations.

\section{References}

1. Adriaansen JJ, Ruijs LE, van Koppenhagen CF, van Asbeck FW, Snoek GJ, van Kuppevelt D. et al. Secondary health conditions and quality of life in persons living with spinal cord injury for at least ten years. J Rehab Med. 2016;48:853-60.
2. Hadjipavlou G, Cortese AM, Ramaswamy B. Spinal cord injury and chronic pain. BJA Educ. 2016;16:264-8.

3. Kirshblum S, Campagnolo DI, DeLisa JA. Spinal cord medicine. Philadelphia: Lippincott Williams and Wilkins; 2002.

4. Finnerup NB. Pain in patients with spinal cord injury. Pain. 2013;154 Suppl 1:S71-6.

5. Piatt JA, Nagata S, Zahl M, Li J, Rosenbluth JP. Problematic secondary health conditions among adults with spinal cord injury and its impact on social participation and daily life. J Spinal Cord Med. 2016;39:693-8.

6. Hill K. Medical marijuana for treatment of chronic pain and other medical and psychiatric problems. JAMA. 2015;313:2474-83.

7. The National Academies Press. The health effects of cannabis and cannabinoids: the current state of evidence and recommendations for research. Washington: The National Academy of Sciences; 2017.

8. Wilsey B, Marcotte T, Tsodikov A, Millman J, Bentley H, Gouaux B. et al. A randomised, placebo-controlled, crossover trial of cannabis cigarettes in neuropathic pain. J Pain. 2008;9:506-21.

9. Hauser W, Finn DP, Kalso E, Krcevski-Skvarc N, Kress HG, Morlion B. et al. European Pain Federation (EFIC) position paper on appropriate use of cannabis-based medicines and medical cannabis for chronic pain management. Eur $\mathrm{J}$ Pain. 2018;22:1547-64.

10. Campbell G, Stockings E, Neielsen S. Understanding the evidence for medical cannabis and cannabis-based medicines for the treatment of chronic non-cancer pain. Eur Arch Psychiatry Clin Neurosci. 2019;269:135-44.

11. Ware MA, Wang T, Shapiro S, Collet J. Cannabis for the management of pain. Assessment of safety study. J Pain. 2015;16:1233-42.

12. Pertwee RG. The diverse CB1 and CB2 receptor pharmacology of three plant cannabinoids: delta-9-tetrahydrocannabinol, cannabidiol and delta-9-tetrahydrocannabivarin. $\mathrm{Br} \quad \mathrm{J}$ Pharmacol. 2008;153:199-215.

13. Hawley LA, Ketchum JM, Morey C, Collins K, Charlifue S. Cannabis use in individuals with spinal cord injury or moderate to severe traumatic brain injury in Colorado. Arch Phys Med Rehabilitation. 2018;99:1584-90.

14. Stillman M, Mallow M, Ransom T, Gustafson K, Bell A, Graves D. Attitudes toward and knowledge of medical cannabis among individuals with spinal cord injury. Spinal Cord Ser Cases. 2019;5:1-6.

15. Stillman M, Capron M, Mallow M, Ransom T, Gustafson K, Bell A. et al. Utilization of medicinal cannabis for pain by individuals with spinal cord injury. Spinal Cord Ser Cases. 2019;5:1-6.

16. Ministry of Health. Misuse of Drugs (Medicinal Cannabis) Amendment Act. 2019; https://http://www.health.govt.nz/our-w ork/regulation-health-and-disability-system/medicines-control/ medicinal-cannabis/misuse-drugs-medicinal-cannabis-a mendment-act. Accessed 16 July 2019.

17. Ministry of Health. Cannabis use 2012/13: New Zealand Health Survey. Wellington: Ministry of Health; 2015.

18. Mucke M, Phillips T, Radbruch L, Petzke F, Haeuser W. Cannabis-based medicines for chronic neuropathic pain in adults. Cochrane Database Syst Rev. 2018;7:1-92.

19. Casey S, Vaughan C. Plant-based cannabinoids for the treatment of chronic neuropathic pain. Medicines. 2018;5:67.

20. Yanes JA, McKinnell ZE, Reid MA, Busler JN, Michel JS, Pangelinan MM. et al. Effects of cannabinoid administration for pain: a meta-analysis and meta-regression. Exp Clin Psychopharmacol. 2019;27:370-82.

21. Berliner J, Collins K, Coker J. Cannabis conundrum. Spinal Cord Ser Cases. 2018;4:1-3. 
22. Graves D. Cannabis shenanigans: advocating for the restoration of an effective treatment of pain following spinal cord injury. Spinal Cord Ser Cases. 2018;4:1-3.

23. Drossel C, Forcheimer M, Meade MA. Characteristics of individuals with spinal cord injury who use cannabis for therapeutic purposes. topics in spinal cord injury. Rehabilitation. 2016;22:3-12.

24. Andresen SR, Biering-Sørensen F, Hagen EM, Nielsen JF, Bach FW, Finnerup NB. Cannabis use in persons with traumatic spinal cord injury in Denmark. J Rehabil Med. 2017;49:152-60.

25. Sadler GR, Lee HC, Lim RSH JF. Recruitment of hard-to-reach population subgroups via adaptations of the snowball sampling strategy. Nurse Health Sci. 2010;12:369-64.

26. Braun V, Clarke V. Using thematic analysis in psychology. Qual Res Psychol. 2006;3:77-101.
27. Braun V, Clarke V. Successful qualitative research: a practical guide for beginners. London: Sage; 2013.

28. Piper BJ, Beals ML, Abess AT, Nichols SD, Martin MW, Cobb CM. et al. Chronic pain patients' perspectives of medical cannabis. Pain. 2017;158:1373-9.

29. Ballantyne JC, Sullivan MD. Intensity of chronic pain-the wrong metric? New Engl J Med. 2015;373:2098-9.

30. Stillman M, Graves DM, New PW, Bryce T, Alexander M. Survey on current treatments for pain after spinal cord damage. Spinal Cord Ser Cases. 2019;5:1-5.

31. Polit DF, Beck CT. Generalisation in quantitative and qualitative research: myths and strategies. Int $J$ Nurs Stud. 2010;47:1451-8.

32. Shenton AK. Strategies for ensuring trustworthiness in qualitative research projects. Educ Inf. 2004;22:63-75. 